\title{
Dynamics of Prolyl Hydroxylases Levels During Disease Progression in Experimental Colitis
}

\author{
Hamid A. Bakshi, ${ }^{1}$ Vijay Mishra, ${ }^{2}$ Saurabh Satija, ${ }^{2}$ Meenu Mehta, ${ }^{2}$ Faruk L. Hakkim, ${ }^{3}$ \\ Prashant Kesharwani, ${ }^{4}$ Kamal Dua, ${ }^{5}$ Dinesh K. Chellappan, ${ }^{6}$ Nitin B. Charbe, ${ }^{7,8}$ \\ Garima Shrivastava, ${ }^{9}$ S. Rajeshkumar, ${ }^{10}$ Alaa A. Aljabali, ${ }^{11}$ Bahaa Al-Trad, ${ }^{12}$ Kavita Pabreja, ${ }^{13}$ \\ and Murtaza M. Tambuwala $\oplus^{1,14}$
}

\begin{abstract}
Hypoxia inducible factor (HIF)-prolyl hydroxylase (PHD) inhibitors are shown to be protective in several models of inflammatory bowel disease (IBD). However, these nonselective inhibitors are known to inhibit all the three isoforms of PHD, i.e. PHD-1, PHD-2 and PHD-3. In the present report, we investigated the associated changes in levels of PHDs during the development and recovery of chemically induced colitis in mice. The results indicated that in the experimental model of murine colitis, levels of both, PHD-1 and PHD-2 were found to be increased with the progression of the disease; however, the level of PHD-3 remained the same in group of healthy controls and mice with colitis. Thus, the findings advocated that inhibitors, which inhibited all three isoforms of PHD could not be ideal therapeutics for IBD since PHD-3 is required for normal gut function. Hence, this necessitates the development of new compounds capable of selectively inhibiting PHD-1 and PHD-2 for effective treatment of IBD.
\end{abstract}

KEY WORDS: prolyl hydroxylases; inflammatory bowel disease; colitis; disease activity index.

\footnotetext{
${ }^{1}$ SAAD Centre for Pharmacy and Diabetes, School of Pharmacy and Pharmaceutical Science, Ulster University, Coleraine, County Londonderry, Northern Ireland BT52 1SA, UK

${ }^{2}$ School of Pharmaceutical Sciences, Lovely Professional University, Phagwara, Punjab, India

${ }^{3}$ Department of Mathematics and Sciences, College of Arts and Applied Sciences, Dhofar University, Salalah, Oman

${ }^{4}$ School of Pharmaceutical Education and Research, Jamia Hamdard (Hamdard University), New Delhi, 110062, India

${ }^{5}$ Discipline of Pharmacy, Graduate School of Health, University of Technology Sydney, Sydney, NSW 2007, Australia

${ }^{6}$ Department of Life Sciences, School of Pharmacy, International Medical University, Bukit Jalil, 57000 Kuala Lumpur, Malaysia

${ }^{7}$ Departamento de Química Orgánica, Facultad de Química y de Farmacia, Pontificia Universidad Católica de Chile, Av. Vicuña McKenna 4860, 7820436 Macul, Santiago, Chile

${ }^{8}$ Sri Adichunchunagiri College of Pharmacy, Sri Adichunchunagiri University, BG Nagar, Karnataka 571418, India
}

${ }^{9}$ Indian Institute of Technology, Delhi, India

${ }^{10}$ Department of Pharmacology, Saveetha Dental College and Hospitals, SIMATS, Chennai, Tamil Nadu 600077, India

${ }^{11}$ Faculty of Pharmacy, Department of Pharmaceutical Sciences, Yarmouk University, Irbid, Jordan

${ }^{12}$ Department of Biological Sciences, Yarmouk University, Irbid, 21163, Jordan

${ }^{13}$ School of Medicine and Public Health, University of Newcastle, Newcastle, Australia

${ }^{14}$ To whom correspondence should be addressed at SAAD Centre for Pharmacy and Diabetes, School of Pharmacy and Pharmaceutical Science, Ulster University, Coleraine, County Londonderry, Northern Ireland BT52 1SA, UK. E-mail: m.tambuwala@ulster.ac.uk 


\section{INTRODUCTION}

Hypoxia-inducible factor (HIF)-prolyl hydroxylases (PHDs) are members of the dioxygenase enzymes family. These enzymes are known to play an important function in intracellular oxygen sensing as well as signalling responses in low oxygen levels (hypoxia). This occurs mainly by the regulation of HIF stability [1-4]. These PHDs consist of three isoforms (PHD-1, PHD-2 and PHD-3), which share mostly similar biochemical characteristics; however, all three isoforms of PHDs are known to have very distinct and tissue-specific expression profiles and, regulation of HIF, which in turn can affect pro-survival signalling pathways, including nuclear factor $\mathrm{KB}$ pathways during inflammation $[1,5-7]$. These PHDs are known to mediate their effect on HIF pathway via hydroxylation of proline residues of the HIF $\alpha$ subunit, which is degraded under normoxic condition. Once it is hydroxylated, $\mathrm{HIF} \alpha$ acts as a target for ubiquitylation by von Hipple Lindau E3 ubiquititin ligase and results in the degradation and ubiquitination of HIF $\alpha$ [8]. The PHDs perform a pivotal function in an adaptive response known to occur during hypoxia via the activation of several pathways of genes expression implicated in support of cell survival, erythropoiesis, angiogenesis and metabolism. These features make PHD an interesting target for therapy [9].

Inflammatory bowel disease (IBD), an idiopathic disease with genetically heterogenous nature, has chronic inflammatory conditions in the gastrointestinal tract (GIT) with severe pathology and limited therapeutic options [1012]. To date, the underlying causes of IBD are not welldefined; however, the fundamental defect in this disease involves damage/injury of the intestinal epithelial barrier resulting in the development and progression of the disease. Hence, targeting the HIF-PHD pathway may be a fascinating area for potential future therapeutics for inflammatory disease.

Various reports employing pharmacological inhibition of hydroxylase enzyme revealed improvisation in a variety of inflammatory conditions including $\operatorname{IBD}[13,15]$. Several researchers reported that pan-hydroxylase inhibitors such as dimethyloxalylglycine (DMOG) have a protective effect in experimental colitis [13-15]. However, the majority of these studies have not identified the actual isoform of PHDs or the effector pathways involved in this protective effect.

Our previous findings suggested that there was an increased PHD-1 level in patients with active ulcerative colitis and genetic loss of PHD-1 was found to be protective in experimental colitis [16]. However, since there are three isoforms of PHDs, it would be of interest for researchers to know how the levels of PHDs change during disease development and recovery in IBD. Thus, to answer this query, the present study has been framed to examine the changes in levels of PHDs (PHD-1, -2 and -3 ) during the inflammation development and its progression in an experimental model of murine colitis.

\section{MATERIALS AND METHODS}

\section{Dextran Sodium Sulphate (DSS)-Induced Colitis}

Colitis was induced in female C57/B16 mice using our previously established protocol $[15,17]$. Briefly, $2.5 \% \mathrm{w} / \mathrm{v}$ dextran sodium sulphate (DSS) was administered in mice via drinking water to induce colitis. Colon tissues were taken at days 2, 3, 4, 5, 6 and 7 from mice exposed to $2.5 \%$ $\mathrm{w} / \mathrm{v}$ DSS in drinking water. The mice of the recovery group were exposed to $2.5 \% \mathrm{w} / \mathrm{v}$ DSS in the drinking water for 5 days and allowed to recover naturally for the next 5 days. On the tenth day, the colon tissue was collected from this group.

\section{Assessment of Prolyl Hydroxylases (PHD) Levels}

The colon tissue lysates were analysed for the level of protein expression of PHD-1, -2 and --3 levels by a Western blotting technique as reported previously [16].

\section{Disease Activity Index (DAI)}

The weight of each animal was recorded on a daily basis. The stool consistency and appearance of faecal blood was monitored. These parameters were converted into the disease activity index (DAI) as illustrated previously [18].

\section{Colon Length}

Each excised colon length was recorded after removal of the faecal matter using a cotton swab dipped in the phosphate-buffer saline (PBS) solution following the previously reported method $[15,18]$.

\section{Statistical Analysis}

The analysis of variance (ANOVA) or Student's $t$ test was employed for statistical comparisons and data was represented as mean \pm standard deviation (mean $\pm \mathrm{SD}$ ). 
a

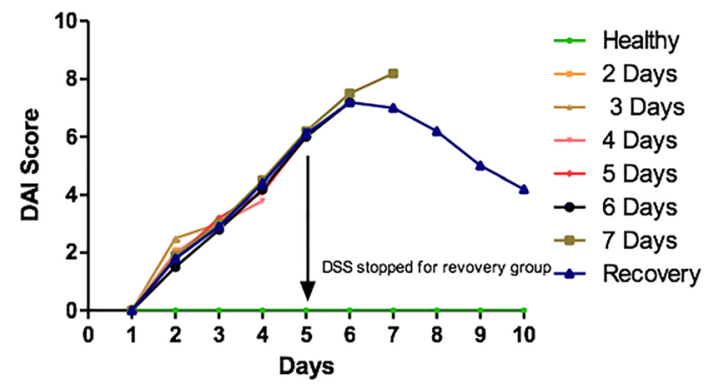

b

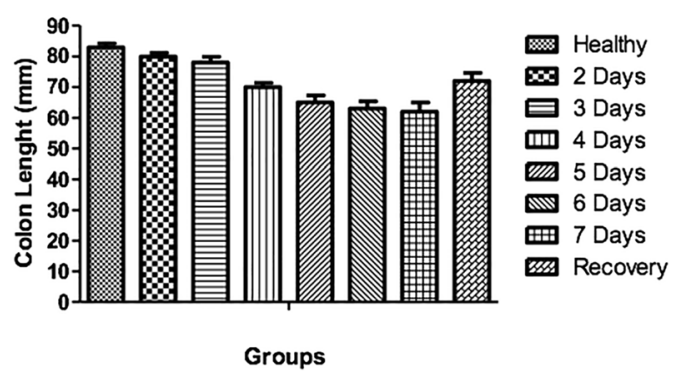

Fig. 1. Changes in disease activity score (DAI) and colon length during active colitis. In a, the composite score of weight loss, stool consistency and blood in faeces during disease progression was represented, and in $\mathbf{b}$, the changes of colon length during active colitis were given. Each control and experimental group contained a minimum of $n=6$ mice.

\section{RESULTS AND DISCUSSION}

The DAI scores indicated that administration of DSS in drinking water caused weight loss, diarrhoea and appearance of blood in faeces, since the DAI steadily raised from day 2 onwards indicating the onset of inflammation from day 2 (Fig. 1a). We have also noted that the colon length of mice progressively decreased as the inflammation progressed (Fig. 1b). This could be due to muscle wasting of the colon tissue during inflammation. Furthermore, both the DAI score and colon length indicated that withdrawal of DSS from the drinking water resulted in tissue recovery and alleviation of the classical symptoms of colitis (Fig. 1).

Our key results indicated that PHD-1 level was found to be lower than that of the healthy control at day 2 and it gradually increased by day 5 (Fig. 2). This event suggested that PHD-1 expression levels initially decreased when inflammation sets in and then progressively increased as

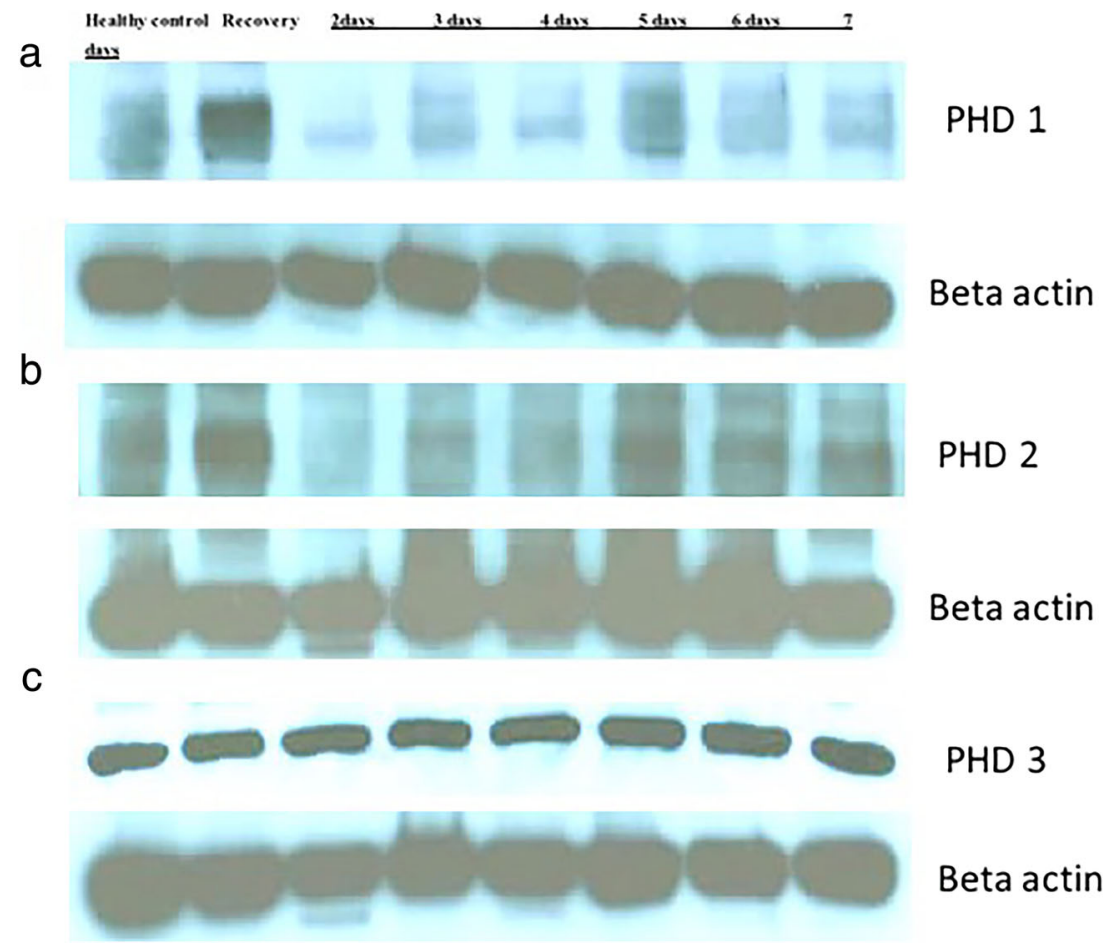

Fig. 2. Colon homogenates assessed for a PHD-1, b PHD-2 and $\mathbf{c}$ PHD-3 protein levels. Each control and experimental group contained a minimum of $n=6$ mice. PHD-1 level was found to be increased with disease progression in murine model of colitis. 
the inflammation was established due to the administration of DSS in the drinking water. The recovery group has shown the highest level of PHD-1 expression in the colon tissue (Fig. 2a), suggesting that once the PHD-1 level is elevated due to inflammation, it does not go down even after the withdrawal of DSS. The PHD-2 levels followed a similar decline trend at day 2 followed by an increase at day 5. In the case of PHD-2, the recovery group also showed the highest levels in the colonic tissue (Fig. 2b). Contrary to this, the levels of PHD-3 were observed to be constant in healthy control, recovery group and during the course of disease progression (Fig. 2c). It suggested that PHD-3 has not involved in the development and progression of inflammation during experimental colitis.

The upregulated PHD-1 in colonic biopsies of IBD patients illustrated the correlation between PHD1 expression and disease severity in patients $[16,19]$. Among PHD1, -2 and -3 , only PHD-1 deficient mice were found to be selectively protected against DSS-induced colitis development advocating an affirmative function of PHD-1 in the management of intestinal epithelial cell apoptosis and preservation of epithelial barrier during intestinal inflammation [16]. The hypothesised link between improved barrier function and consequent protection against the colitis development was also supported by studies performed in other models, indicating an antiapoptotic effect of pharmacologically inhibited hydroxylase enzyme [20-22].

\section{CONCLUSION}

These results suggest that PHD-3 isoform is expressed in healthy as well as the diseased colon tissue, indicating that PHD-3 is required for the normal function of the gut. However, PHD-1 and -2 expression levels fall when inflammation is initiated and gradually increases during the progression of inflammation and is observed to be the highest in chronic stages. The recovery group indicates that once the agent causing inflammation of the colon, i.e. DSS is removed from the drinking water, these mice show signs of recovery (Fig. 1); however, the increased levels of PHD-1 and -2 are not reduced to normal level, which suggest that PHD-1 and -2 are involved in chronic inflammatory stages of the disease. These findings advocate that inhibition of all PHDs using a non-selective prolyl hydroxylases inhibitor may not be the best strategy towards the development of new therapeutics for IBD. Hence, strategies to selectively target PHD-1 and -2 are warranted leading to an improved therapeutic advancement in the field of IBD treatment.

\section{COMPLIANCE WITH ETHICAL STANDARDS}

All animal experiments were performed under UK Home Office personal and project licence.

Conflict of Interest. The authors declare that they have no conflict of interest.

Open Access This article is distributed under the terms of the Creative Commons Attribution 4.0 International License (http://creativecommons.org/licenses/by/4.0/), which permits unrestricted use, distribution, and reproduction in any medium, provided you give appropriate credit to the original author(s) and the source, provide a link to the Creative Commons license, and indicate if changes were made.

\section{REFERENCES}

1. Kaelin, W., and P. Ratcliffe. 2008. Oxygen sensing by metazoans: the central role of the HIF hydroxylase pathway. Molecular Cell 30: 393-402.

2. Schipani, E., C. Maes, G. Carmeliet, and G.L. Semenza. 2009. Regulation of osteogenesis angiogenesis coupling by HIFs and VEGF. Journal of Bone and Mineral Research 24: 1347-1353.

3. Semenza, G. 2009. Involvement of oxygen-sensing pathways in physiologic and pathologic erythropoiesis. Blood 114: 2015.

4. Semenza, G. 2008. Hypoxia inducible factor 1 and cancer pathogenesis. IUBMB Life 60: 591-597.

5. Cummins, E.P., E. Berra, K.M. Comerford, A. Ginouves, K.T. Fitzgerald, F. Seeballuck, C. Godson, J.E. Nielsen, P. Moynagh, J. Pouyssegur, and C.T. Taylor. 2006. Prolyl hydroxylase-1 negatively regulates IkappaB kinase-beta, giving insight into hypoxia-induced NFkappaB activity. Proceedings of the National Academy of Sciences of the United States of America 103: 18154-18159.

6. Chan, D., T. Kawahara, P. Sutphin, et al. 2009. Tumor vasculature is regulated by $\mathrm{PHD} 2-$ mediated angiogenesis and bone marrowderived cell recruitment. Cancer Cell 15: 527-538.

7. Fu, J., and M.B. Taubman. 2010. Prolyl hydroxylase EGLN3 regulates skeletal myoblast differentiation through an NF-kappaB-dependent pathway. The Journal of Biological Chemistry 285: 8927-8935.

8. Oliver, K.M., C.T. Taylor, and E.P. Cummins. 2009. Hypoxia. Regulation of NFkappaB signalling during inflammation: the role of hydroxylases. Arthritis Research \& Therapy 11: 215.

9. Fraisl, P., J. Aragones, and P. Carmeliet. 2009. Inhibition of oxygen sensors as a therapeutic strategy for ischaemic and inflammatory disease. Nature Reviews. Drug Discovery 8: 139-152.

10. Braus, N., and D. Elliott. 2009. Advances in the pathogenesis and treatment of IBD. Clinical Immunology 132: 1-9.

11. Rutgeerts, P., S. Vermeire, and G. Van Assche. 2009. Biological therapies for inflammatory bowel diseases. Gastroenterology 136 : 1182-1197.

12. Imielinski, M., R.N. Baldassano, A. Griffiths, et al. 2009. Common variants at five new loci associated with early-onset inflammatory 
bowel disease. Nature Genetics 41(12): 1335-1340. https://doi.org/ 10.1038/ng. 489 .

13. Cummins, E., F. Seeballuck, S. Keely, et al. 2008. The hydroxylase inhibitor dimethyloxalylglycine is protective in a murine model of colitis. Gastroenterology 134: 346-348.

14. Robinson, A., S. Keely, J. Karhausen, M.E. Gerich, G.T. Furuta, and S.P. Colgan. 2008. Mucosal protection by hypoxia-inducible factor (HIF) prolyl hydroxylase inhibition. Gastroenterology 134: 145.

15. Tambuwala, M.M., M.C. Manresa, E.P. Cummins, V. Aversa, I.S. Coulter, and C.T. Taylor. 2015. Targeted delivery of the hydroxylase inhibitor DMOG provides enhanced efficacy with reduced systemic exposure in a murine model of colitis. Journal of Controlled Release 217: 221-227.

16. Tambuwala, M.M., E.P. Cummins, C.R. Lenihan, J. Kiss, M. Stauch, C.C. Scholz, P. Fraisl, F. Lasitschka, M. Mollenhauer, S.P. Saunders, P.H. Maxwell, P. Carmeliet, P.G. Fallon, M. Schneider, and C.T. Taylor. 2010. Loss of prolyl hydroxylase-1 protects against colitis through reduced epithelial cell apoptosis and increased barrier function. Gastroenterology 139: 2093-2101.

17. Khan, M.N., M.E. Lane, P.A. McCarron, and M.M. Tambuwala. 2018. Caffeic acid phenethyl ester is protective in experimental ulcerative colitis via reduction in levels of pro-inflammatory mediators and enhancement of epithelial barrier function. Inflammopharmacology 26(2): 561-569. https://doi.org/10.1007/ s10787-017-0364-x.

18. Cooper, H.S., S.N. Murthy, R.S. Shah, et al. 1993. Clinicopathologic study of dextran sulfate sodium experimental murine colitis. Laboratory Investigation 69: 238-249.
19. Van Welden, S., et al. 2013. Differential expression of prolyl hydroxylase 1 in patients with ulcerative colitis versus patients with Crohn's disease/infectious colitis and healthy controls. Journal of Inflammation (London, England) 10: 36.

20. Liu, X.B., J.A. Wang, M.E. Ogle, and L. Wei. 2009. Prolyl hydroxylase inhibitor dimethyloxalylglycine enhances mesenchymal stem cell survival. Journal of Cellular Biochemistry 106: 903-911.

21. Lomb, D.J., J.A. Straub, and R.S. Freeman. 2007. Prolyl hydroxylase inhibitors delay neuronal cell death caused by trophic factor deprivation. Journal of Neurochemistry 103: 1897-1906.

22. Nangaku, M., Y. Izuhara, S. Takizawa, T. Yamashita, Y. FujiiKuriyama, O. Ohneda, M. Yamamoto, C. van Ypersele de Strihou, N. Hirayama, and T. Miyata. 2007. A novel class of prolyl hydroxylase inhibitors induces angiogenesis and exerts organ protection against ischemia. Arteriosclerosis, Thrombosis, and Vascular Biology 27: 2548-2554.

Publisher's Note Springer Nature remains neutral with regard to jurisdictional claims in published maps and institutional affiliations. 